\title{
Dual Connectivity in Heterogeneous Small Cell Networks with mmWave Backhauls
}

\author{
Wooseong Kim \\ Department of Computer Engineering, Gachon University, 1342 Seongnam-ro, Kyeonggi, Republic of Korea
}

Correspondence should be addressed to Wooseong Kim; wooseong@gachon.ac.kr

Received 27 May 2016; Revised 3 September 2016; Accepted 8 September 2016

Academic Editor: Laurence T. Yang

Copyright (C) 2016 Wooseong Kim. This is an open access article distributed under the Creative Commons Attribution License, which permits unrestricted use, distribution, and reproduction in any medium, provided the original work is properly cited.

Ultradense Network (UDN) with small cells is a key feature to begin a new era of 5G communication, which provides higher data rate, and accommodate explosive mobile traffic. Recently, mmWave-based wireless backhauls accelerate deployment of the UDN by reducing cost of fiber-optic cabling to small cells. The small cells can deliver user data to macro enhanced NodeBs (eNBs) using multihop relay in wireless backhaul mesh that consists of small and macro cell eNBs connected by the mmWave links. For such a heterogeneous small cell network (HetNet), 3GPP introduced dual connectivity (i.e., dual connections to macro and small cell eNBs), which is an attractive standard feature to manage user mobility and network access in the small cells. In this paper, we exploit dual connectivity scheme in a HetNet with the mmWave-based backhaul mesh which introduces two main challenges for throughput maximization, multihop routing from small to macro cell, and selection of a small cell eNB for user equipment (UE). We establish an optimization model and find an optimal solution in terms of throughput and fairness using an IBM CPLEX solver. Additionally, we propose a heuristic algorithm for complexity reduction and compare it with the optimal results in evaluation.

\section{Introduction}

Fast growing mobile traffic demands small cell deployment at urban hot spots, which can increase effectively network capacity by cell densification and data rate with proximity to user equipment (UE). 5G projects $[1,2]$ address Ultradense Network (UDN) with the small cells as a key technology to accommodate 10-100x increasing traffic. In the 5G, different radio access technologies (RATs) and sizes of the small cells lead to heterogeneity in aspect of network architecture and spectral usages [3].

Recently, mmWave technologies receive much attention in mobile communication area, which allows users to utilize superwide bands (e.g., $2.1 \mathrm{GHz}$ bands at $60 \mathrm{GHz}$ versus $20 \mathrm{MHz}$ at $2.4 \mathrm{GHz}$ ) with light license or without license to achieve Giga-bit rate data communication. IEEE 802.15.3 Task Group 3c (TG3c) [4], IEEE 802.11ad [5], and WiGig [6] alliance have made great effort to develop Giga-bit local area communication that provides Giga-bit links among mobile devices and home appliances and standardize physical and protocol technologies.

Recent measurement campaigns in urban areas and demonstrations by manufacturers, universities, and network operators show that the mmWave communication can be applicable to wireless cellular networks [7-9]. The mmWave especially is attractive for backhaul or fronthaul of heterogeneous small cell networks (HetNets). Up to now, the backhaul or fronthaul between macro and small cell enhanced NodeBs (i.e., MeNB and SeNB) is established using fiber-optic cables, which is costly and unscalable. In contrast, mmWave wireless backhaul or fronthaul carries mobile traffic flexibly using more or less spectrum and is easy to deploy and manage compared to wire backhaul or fronthaul. Unfortunately, the mmWave backhauls almost require Line-of-Sight (LoS) between a sender and a receiver due to severe penetration loss and reflection. In addition, higher path loss due to oxygen absorption and rain effect than conventional cellular spectrum limits backhaul range between two eNBs. Accordingly, mmWave-based backhauls from an SeNB to an MeNB or gateway are created by multiple LoS links between eNBs, by which SeNBs and MeNBs form a multihop wireless mesh network (WMN) and the eNBs deliver user equipment (UE) data by multihop routing in the mesh network.

Intertier operation between the macro and small cells in HetNets is necessary to manage network resources and UEs 
effectively. Legacy LTE networks provide a Carrier Aggregation (CA) technique which combines scattered carriers on different frequencies of those macro and remote small cells within a single eNB. Herein, UEs are controlled by the macro cell in terms of network access, handover, security, and so forth, while user data are delivered by both cells simultaneously. However, this CA is difficult to apply in the mmWave-based backhaul mesh network because multihop delay in the backhaul causes asynchronous transmission in the macro and small cells.

Instead, dual connectivity is introduced in 3GPP Rel-12 specification to support separate connections to the MeNB and SeNB (we abuse these acronyms to indicate cell size of an eNB instead of a Master eNB and Secondary eNB in 3GPP specification) for UE, which allows asynchronous transmission in the dual connections for two bearers or split bearers in a higher layer (i.e., Packet Data Convergence Protocol (PDCP) layer) that has a function to reorder out-of-ordered packets due to backhaul delay. In the dual connectivity, the MeNB also controls UEs like in the CA which decides an SeNB of UE for dual connectivity based on UE measurement report. To say, the MeNB selects one of SeNBs of small cells detected by the UE and commands the SeNB and UE to make a secondary connection.

The dual connectivity in the HetNets with mmWavebased backhauls introduces two main challenges to maximize network throughput; one is SeNB selection considering cell capacity and the other is multihop route selection in the mmWave-backhaul mesh. In this study, we establish a mathematical model of the dual connectivity in mmWave-backhaul HetNets and solve the optimization problem using IBM CPLEX. Due to complexity of the problem, we propose an alternative heuristic algorithm for the problem and perform simulation to compare both approaches.

The remainder of this paper is organized as follows: we introduce background knowledge with dual connectivity and mmWave communication in Section 2. Section 3 describes a mmWave-backhaul HetNet architecture and a system model with the two major challenges. We present preliminary performance evaluation of simple topologies and explain a proposed algorithm and performance comparison in Section 4. In Section 5, we show previous works in heterogeneous small cell networks and mmWave communication. We conclude the paper in Section 6.

\section{Heterogeneous Networks}

Increasing smart handheld devices and associated traffic accelerate small cell deployment which can increase network capacity, per-user throughput, and QoS. The small cells can also offload congested macro cells, improve received signal quality at macro cell edge, and extend cell coverage [10].

Figure 1 shows small cell deployment scenarios. Coexistence of existing macro and newly deployed small cells (e.g., micro-, pico-, and femtocells) forms two-layer (tier) wireless heterogeneous networks (HetNets) [11-14]. The HetNets can use the same or different frequencies for the macro and small cells. For example, the macro and small cells can use the same frequency with a Frequency-Division Duplexing (FDD), for example, at $1.9 \mathrm{GHz}$ and $2.1 \mathrm{GHz}$ for up- and downlinks in Figure 1(a), or the small cells use a different frequency, for example, at $2.6 \mathrm{GHz} \mathrm{FDD}$ or $3.5 \mathrm{GHz} \mathrm{TDD}$ spectrum in Figure 1(b). Within the macro cell, small cells form dense clusters with minimum $20 \mathrm{~m}$ intersite distance (ISD) at urban hot spots $[11,13,14]$. Also, the small cell clusters can be deployed without the macro cell as in Figure 1(c). Regardless of the frequency, small cells can be installed indoor or outdoor.

Standard organizations such as IEEE and 3GPP make an effort to publish standards for operation and management of the small cell networks. In 3GPP Rel-11 standard for LTEAdvance (LTE-A), Carrier Aggregation (CA) of macro and remote small cells in HetNets was introduced, which allows a central macro eNB (MeNB) to schedule radio resources of those two cells dynamically for user equipment (UE) $[10,12]$. HetNet mobility mechanism between the small cells has been explored to prevent service disruption during handover [15]. In 3GPP Rel-12, the 3GPP released standards about new small cell enhancement (SCE) that cope with higher modulation scheme (e.g., 256 QAM), small cell on-off and discovery for power saving, radio interface based cell synchronization, and dual connectivity in the two-tier HetNets [11, 14].

2.1. Dual Connectivity. Dual connectivity enables UE to have two separate connections to an MeNB of macro cells and an SeNB of small cells, simultaneously. The CA defined in 3GPP Rel-10/11 also allows UE to have multiple connections to multiple serving cells (i.e., the macro and small cells) to increase per-user throughput. However, those serving cells for the CA should belong to a single eNB that can have multiple serving cells by sectors and operational frequencies. For example, an eNB has 6 serving cells with 2 carrier frequencies and 3 sectors. In contrast, the serving cells belong to different eNBs (i.e., MeNB and SeNB) in the dual connectivity. For this reason, the Rel-10/11 CA and dual connectivity are called as intra- and inter-eNB CA, respectively.

The dual connectivity can improve not only the peruser data throughput, but also mobility robustness. UEs can receive data of a bearer via the dual connections simultaneously. And UE can separate transmission of control messages and user data to MeNB and SeNB, respectively (called as Control Plane (CP) and User Plane (UP) split). Here, UE does not need handover between small cells in the UP while the UE is connected to the overlying MeNB in the CP.

Dual connectivity is a fascinating technique for small cell deployment using stand-alone SeNBs compared to the Rel$11 \mathrm{CA}$ where synchronous transmission in macro and small cells is required. Thus, fiber-optic cables between an MeNB and remote radio headers (RRHs) for small cells are typically installed. In contrast, the dual connectivity allows relatively long backhaul latency (e.g., more than $20 \mathrm{msec}$ ) between two serving cells, which requires a packet reordering function in a higher layer for bearer split.

2.1.1. Control Signal Flow. In the dual connectivity, an MeNB controls underlying SeNBs of small cells for UE (i.e., adding 

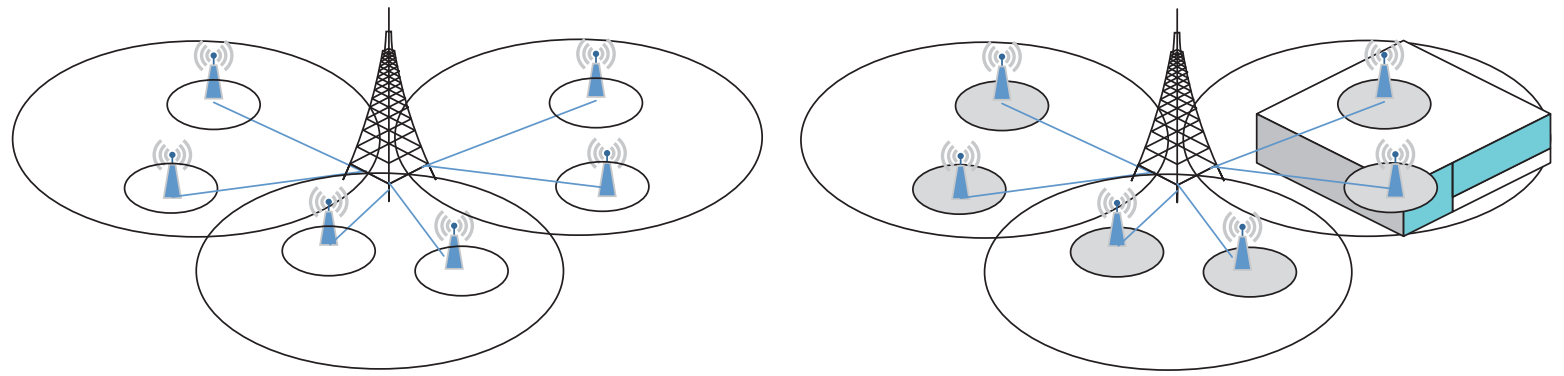

Frequency $1(F 1)$

Frequency 2 (F2)

(a) Intrafrequency eNB CA

(b) Interfrequency eNB CA

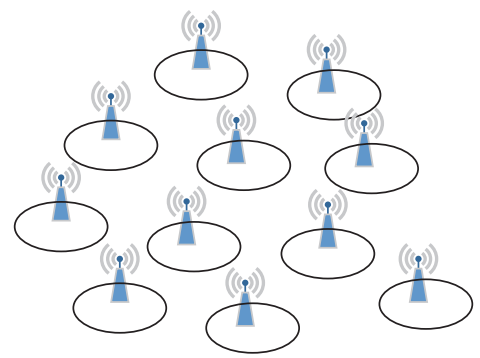

(c) Only small eNBs

FIGURE 1: Small cell deployment scenarios in TR 36.872 [11]. (a) Intrafrequency eNB CA: SeNBs operate on the same carrier frequency as an MeNB (i.e., cochannel deployment). Due to backhaul delay, collaborative scheduling to avoid mutual interference between them is challenge. (b) Interfrequency eNB CA: SeNBs tune to different carrier frequencies like $3.5 \mathrm{GHz}$ suitable to small cells. SeNBs create several small cell clusters within the macro coverage. (c) SeNBs without CA: only SeNBs without the overlaid MeNB are deployed.

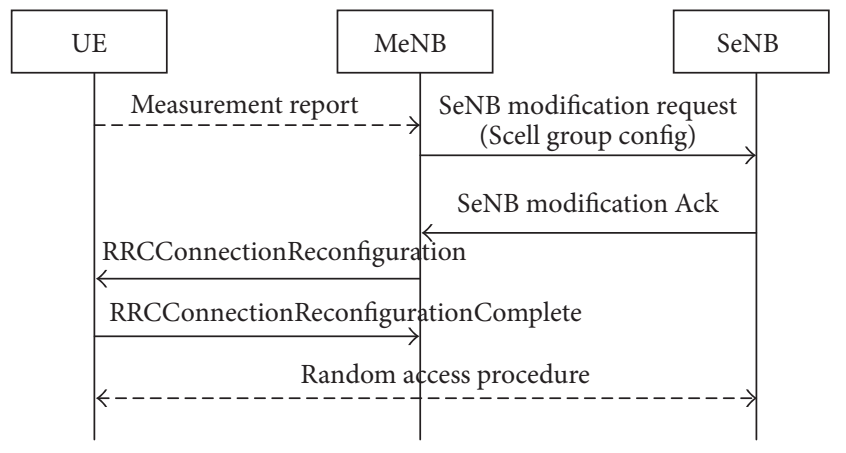

(a) MeNB initiated SCell change

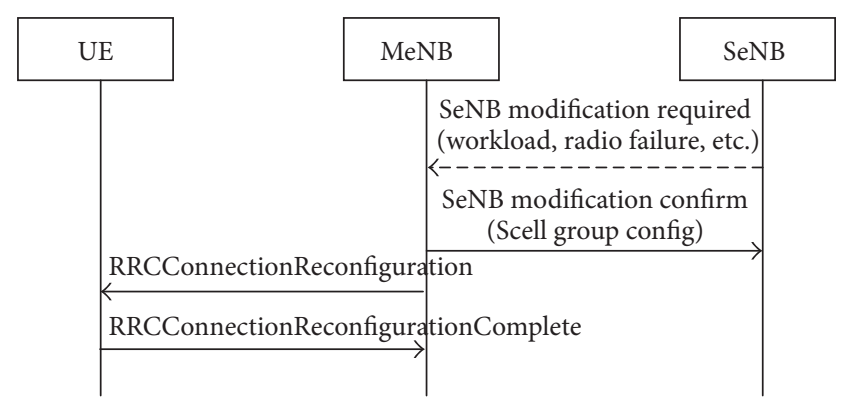

(b) SeNB initiated SCell modification

FIGURE 2: SeNB configuration procedure in dual connectivity.

or releasing serving cells of the SeNBs) via a backhaul connection, $X_{2}$ between the MeNB and SeNB [14]. Thus, only the MeNB has a Radio Resource Control (RRC) function for the UE in the dual connectivity. Otherwise, distributed UE contexts about radio resource configuration, network connection, and device capability require painful interoperation to synchronize the contexts between the MeNB and SeNB.

For dual connectivity establishment, UE periodically reports measurement on small cells of the SeNBs to the MeNB. If those small cells are qualified for serving the UE, the MeNB commands the UE to open a new connection to the SeNB and makes the corresponding SeNB assign radio resources for the connection at the same time. Then, the MeNB divides UE data in the dual connections adaptively based on traffic load and wireless channel quality.

Signal flows for addition, modification, and release of the SeNB's Secondary Cells (SCells) are present in 3GPP Rel12 specification. Figure 2 shows details of two exemplary message flows for small cell addition or release by MeNB or SeNB. In Figure 2(a), an MeNB initiates a procedure to add or release SCells of an SeNB upon receiving the MeasurementReport from UE. Additionally, this procedure could be initiated when the MeNB detects change in macro cell load (e.g., congestion in the macro cell) or receives the 


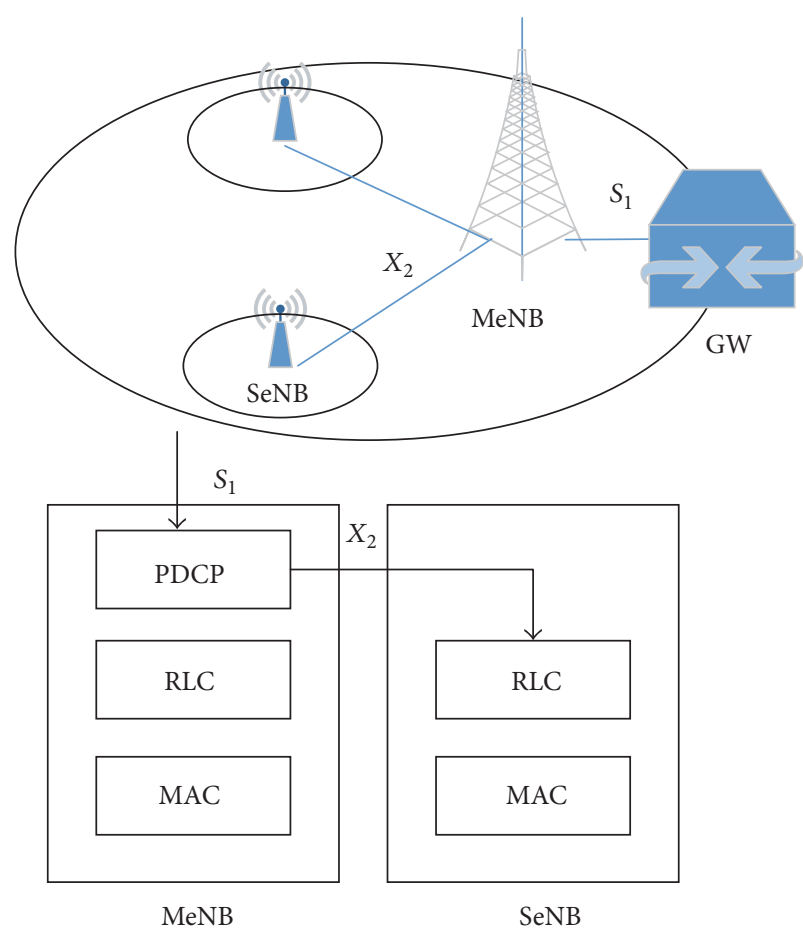

(a) RAN split

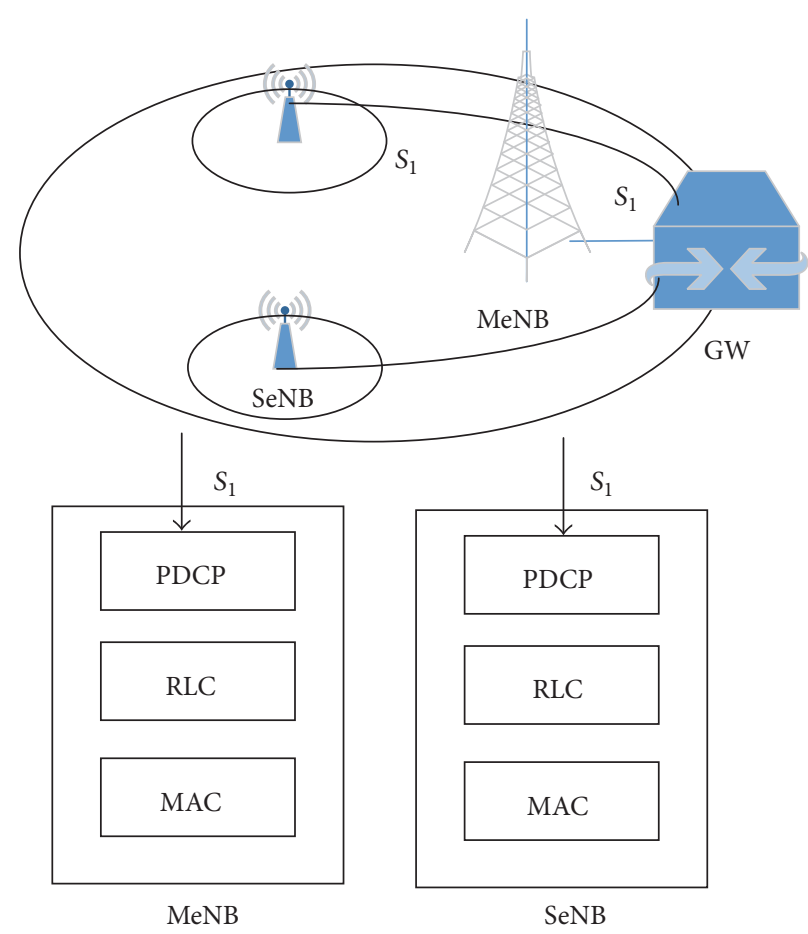

(b) CN split

FIGURE 3: RAN split versus CN split.

ResourceStatusUpdate from the SeNB; the SeNB status may trigger the MeNB to add or release SCells of SeNBs according to utilization.

When sending SCG-ConfigInfo to the SeNB for SCell configuration, the MeNB provides the identifier of the SCell to be added or released (e.g., frequency and physical cell ID (PCI)) and any restriction to be considered (e.g., UE capabilities). And then, the SeNB decides its configuration within the restriction. Alternatively, the MeNB could inform the SeNB of the current UE configuration together with its capabilities for reference. Based on this information the SeNB configures by itself without exceeding UE capabilities. After the SeNB decides SCell Group (SCG) configuration, the SeNB forwards results to the MeNB by using SCellToAddModList or SCellToReleaseList for adding or releasing, respectively. The MeNB forwards the SCell configuration to the UE using a message container in the RRCConnectionReconfiguration.

Figure 2(b) shows SeNB initiated SCell modification procedure by which the SeNB modifies its SCell configuration such as Physical Uplink Control Channel (PUCCH) configuration. The procedure is similar with the MeNB initiated modification.

2.1.2. User Data Flow. For user data flows, two different architectures, Radio Access Network (RAN) split and Core Network (CN) split, can be considered as shown in Figure 3. In the RAN split, an SeNB comes out from an MeNB via $X_{n}\left(X_{2}\right)$ backhaul. In the CN split, the SeNB is directly connected to a serving gateway (S-GW). Therefore, bearer split in the RAN split increases peak data rate of a single bearer by concurrent transmissions in the dual connections, but in the CN split the SeNB serves another bearer direct from the S-GW. This direct backhaul to the CN can carry large traffic from the SeNBs compared to the RAN split. However, the CN split causes control overhead in the CNs with frequent handover among SeNBs (e.g., data path switch). Consequently, the RAN split architecture is more scalable and flexible for ultradense small cell networks.

In contrast to Rel-10/11 CA where user data are multiplexed to multiple carriers at a MAC layer, a bearer split is handled at a Packet Data Convergence Protocol (PDCP) layer in the RAN split. The MAC and Radio Link Control (RLC) layers should be located independently on each eNB as shown in Figure 3(a) in order to segment a packet adaptively based on transport block (TB) size that is determined by channel quality. Otherwise, backhaul latency hinders adaptive scheduling and packetizing user data based on the physical channel status.

2.2. mmWave Backhaul. With dense small cells in $5 \mathrm{G}$ cellular networks, it is costly to connect all SeNBs of the small cells to networks using fiber-optic cables in terms of deployment time and wiring expense. Wireless backhaul is alternative for the small cell deployment which is cost-effective, flexible, and easier to deploy compared to the fiber-optic cables.

Recently, 5G mobile communication will envisage 10100x higher data rate than current rate for each user in year 2020 (e.g., 1-10 Gbps) [2, 16]. mmWave communication receives much attention for access or backhaul networks, which can provide Giga-bit data rate with huge bandwidth 
available in above $6 \mathrm{GHz}$ spectrum. For instance, $60 \mathrm{GHz}$ band and E-band $(7176 \mathrm{GHz}$ and $8186 \mathrm{GHz})$ have 9 and $10 \mathrm{GHz}$ available bandwidth, respectively [17].

Several standards are already defined for Giga-bit rate data communication in wireless LAN or PAN using the $60 \mathrm{GHz}$ bands, such as ECMA-387 [18], IEEE 802.15.3 Task Group 3c (TG3c) [4], and IEEE 802.11ad [5]. IEEE 802.11ad specifies a physical and MAC layer in the $60 \mathrm{GHz}$ to support high data rate applications such as remote display of Ultrahigh Definition (UHD), device-to-device connectivity, and the Internet access. 802.11ad and WiGig [6] provide approximately $6 \mathrm{Gbps}$ using a single $2.16 \mathrm{GHz}$ channel; there are 4 channels at $57-66 \mathrm{GHz}$. IEEE $802.15 .3 \mathrm{c}$ also specifies a physical and MAC layer of a piconet architecture for WPANs.

For $5 \mathrm{G}$ mobile networks, mmWave communication is researched popularly as measurement campaigns in urban areas conducted at $28,38,60$, and $73 \mathrm{GHz}$ show possibility of mmWave use for mobile communication [7-9].

The mmWave-backhaul links for small cells should be deployed in Line-of-Sight (LoS) because the mmWave bands have high path loss (e.g., higher oxygen absorption and raining effect) and low penetration rate compared to microwave spectrum that is typically used for wireless cellular communications. For instance, the oxygen absorption is $15 \mathrm{~dB} / \mathrm{km}$ and the normal rainfall attenuation (i.e., $50 \mathrm{~mm} / \mathrm{h}$ ) is $17 \mathrm{~dB} / \mathrm{km}$ at $60 \mathrm{GHz}$. In addition, LoS for the backhaul links is not easy to acquire in urban environment that includes various height buildings and structures. Therefore, multihop relays using short-distance (e.g., 100-200 m) and highly capable links are more effective than using a single long-distance link for wireless backhauls. The multihop routing scheme finds an optimal path to small cells for UE traffic in the backhaul meshes, which however can introduce additional delay for packet delivery compared to the single-hop link.

The mmWave-backhaul links can use different bands and directional antenna to avoid interference from other links. On the other hand, the same band can be used for point-tomultipoint communication and cost efficiency (e.g., limited number of transceivers) using TDM scheme. Reference [19] introduces a HetNet architecture using mmWave communication for access and backhaul networks and multihop routing from UEs to MeNBs in the backhaul meshes. Therein, a frame-based TDD and TDM scheme is explored for radio resource allocation in the access and backhaul links, which increases spatial reuse of mmWave bands for small cell clusters. Also, in [20], a TDM-based scheduling for in-band access and backhaul links is investigated to support point-tomultipoint and non-LoS communication.

Recently, 3GPP started discussion for 5G standard which includes the mmWave-based wireless backhauls and multihop relay for HetNets [21, 22].

\section{System Model}

In this section, first we introduce an architecture of HetNets with mmWave-backhaul as evolution to $5 \mathrm{G}$ wireless networks. In the HetNets, we establish an optimization model

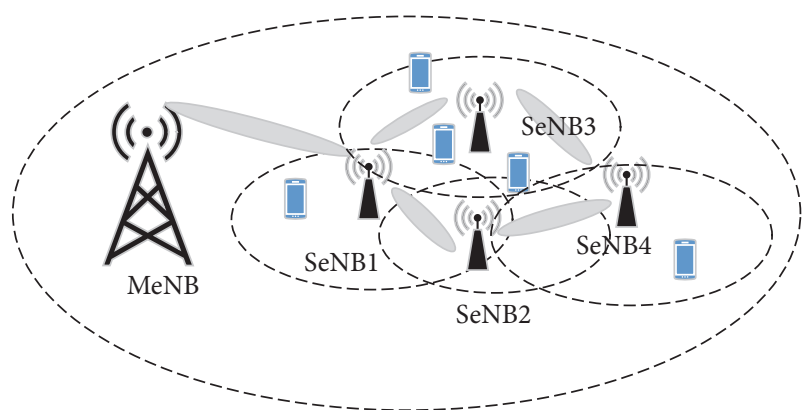

Figure 4: A HetNet of an MeNB and SeNBs with multihop mmWave backhauls.

of SeNB selection and multihop routing from the SeNB to an MeNB for dual connectivity.

3.1. Network Architecture. The $5 \mathrm{G}$ HetNet that consists of an MeNB and multiple SeNBs for small cells linked by mmWave backhauls in urban hot spots is illustrated in Figure 4. The SeNBs use above $60 \mathrm{GHz}$ spectrum for $X_{2}$ backhauls to connect them to the MeNB or adjacent SeNBs. The SeNBs can reach the MeNB using multihop relays if they do not have a direct backhaul link to the MeNB. For example, the MeNB sends UEs' downlink data through the SeNB1 and SeNB2 if the UEs are attached to the SeNB4 in Figure 4.

The MeNB can select an optimal multihop route for the $X_{2}$ interface between the MeNB and SeNB, which maximizes UE throughput. For example, the SeNB1 has two paths to deliver data to UEs in SeNB4, via SeNB2 or SeNB3 for downlink transmission from the MeNB in Figure 4. The SeNB3 has more associated UEs (e.g., 3 UEs) rather than the SeNB2 (e.g., 0 UEs), which implies that the backhaul between the SeNB1 and SeNB3 may have more traffic than the SeNB2. Accordingly, the SeNB1 can select the SeNB2 as a next hop. But it can be varying with the amount of associated UE traffic. Thus, we need to investigate the optimal route selection based on given all UE flows in backhaul mesh networks.

Another problem is UE association in a small cell cluster. Previously, UEs select an eNB based on measurement of signal strength and quality such as Reference Signal Received Power (RSRP) and Reference Signal Received Quality (RSRQ). In urban area, UEs can have multiple SeNBs of which signal quality is similar to each other within a small cell cluster.

Figure 5 depicts a simple example of the SeNB association problem. The UE3 has two candidate SeNBs for secondary serving cells, SeNB1 and SeNB2. But those SeNBs have different number of associated UEs; SeNB1 has 2 UEs and SeNB2 has 1 UE except the UE3. Thus, the UE3 seems profitable to associate with the SeNB2 rather than the SeNB1. However, it also depends on the amount of those UE traffic; UE4 traffic can be more than total traffic of UE1 and UE2.

3.2. Optimization Model. In this section, we establish an optimization model to find an optimal solution of dual connectivity in HetNets with mmWave backhauls, which 


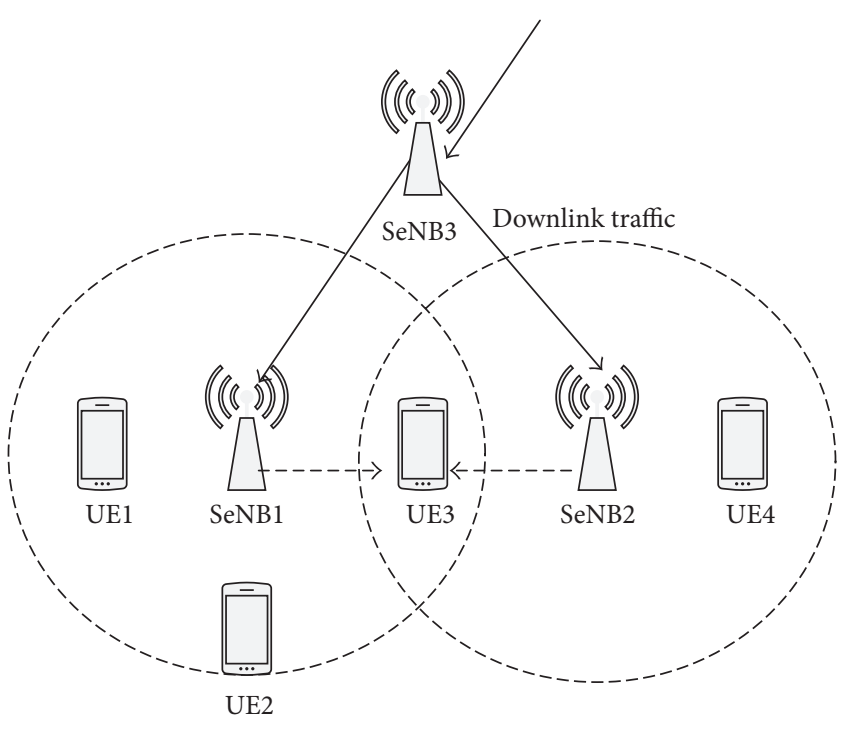

FIGURE 5: SeNB selection based on backhaul traffic.

provides SeNB selection and a multihop route for a backhaul. Parameters and variables for this optimization model are shown in Notations. Main objective of this system model is maximizing total UE throughput with fairness consideration as shown in the following:

$$
\max U\left(F^{u}\right)
$$

The utility function $U$ is defined as follows:

$$
\begin{aligned}
& U\left(F^{u}\right)=\gamma+\kappa \sum_{u \in U} F^{u}, \\
& \gamma-F^{u} \leq 0, \quad \forall u \in U,
\end{aligned}
$$

where $\gamma$ is minimum data rate for UE and $\kappa$ is a value for fairness among UEs. The utility function considers maxmin fairness with small $\kappa$ value, for example, $1 e-3$, and proportional fairness with a big $\kappa$ value, for example, $1 e+3$.

UE flows follow conservation rule in $(3), s(u)$ indicates a source $\mathrm{eNB}$ of $\mathrm{UE}$, and $u$ and $d(u)$ are a sink eNB of the UE:

$$
\begin{aligned}
& \sum_{(i, j)} f_{i j}^{u}-\sum_{(j, i)} f_{j i}^{u}=F^{u}, \quad \forall i, j \in N, \\
& F^{u}= \begin{cases}f_{s}^{u}, & \text { if } i=s(u), \\
-f_{d}^{u}, & \text { if } i=d(u), \\
0, & \text { otherwise, }\end{cases} \\
& f_{i j}^{u} \geq 0, \quad u \in U, i, j \in \mathcal{N} .
\end{aligned}
$$

UE flow rate has link capacity constrains in access and backhaul links. First, the UE flow rate on the access link should be less than achievable link capacity of the UE as shown in (4), which is determined by SINR and bandwidth $B_{l}$ of the link (in simulation, the link capacity for the UE is calculated by Modulation Coding Scheme (MCS) level of allocated PRBs, not by the Shannon equation because of throughput gap):

$$
\begin{aligned}
& f_{l}^{u} \leq C_{l}, \quad l \in \mathscr{L}_{\mathscr{A}}, \\
& C_{l}=B_{l} \cdot \log \left(1+\mathrm{SINR}_{l}\right) .
\end{aligned}
$$

The bandwidth is decided by a number of PBRs assigned for the UE. The number of PRBs is limited by a system bandwidth, for example, $50 \mathrm{PRBs}$ for $10 \mathrm{MHz}$, which introduces the following constraints:

$$
\begin{aligned}
B_{l} & =\mathrm{PRB}_{l}, \quad \forall l \in \mathscr{L}_{\mathscr{A}} \\
\sum_{(i, u) \in \mathscr{L}_{\mathscr{A}}} \mathrm{PRB}_{(i, u)} & \leq N_{\mathrm{PRB}}, \quad \forall i \in \mathcal{N} .
\end{aligned}
$$

Next, aggregated UE flows in a backhaul link should be less than the backhaul capacity:

$$
\sum_{u} f_{l}^{u} \leq C_{l}, \quad l \in \mathscr{L}_{\mathscr{B}}
$$

TDD and TDM-based slot scheduling of a single band for multiple backhaul links [19, 20] follows constraint (7). Herein the backhaul capacity has to be shared in time by adjacent backhaul links, $(m, n) \in I(i, j)$ of a link $(i, j)$. Furthermore, the set $I$ is varying according to number of bands (i.e., channels) and channel assignment used for the backhaul links. For instance, if all backhaul links use separate bands like FDM, the $I$ can be empty:

$$
\sum_{u \in U} \frac{f_{i j}^{u}}{c_{i j}}+\sum_{u \in U} \sum_{(m n) \in I((i j))} \frac{f_{m n}^{u}}{c_{m n}} \leq 1, \quad \forall i, j, m, n \in \mathcal{N} .
$$

For dual connectivity, constraints for separate connections to MeNB and SeNB are defined as

$$
\begin{aligned}
& \sum_{(i, u)} a_{(i, u)}^{u}=1, \quad i \in \mathscr{M}, \\
& \sum_{(i, u)} a_{(i, u)}^{u}=1, \quad i \in \mathcal{S},
\end{aligned}
$$

where $a_{i u}^{u}$ is a binary variable to indicate whether the UE $u$ is associated with the eNB $i . a_{i u}^{u}=1$ if the UE $u$ has a downlink carrier at the eNB $i$ and 0 , otherwise:

$$
f_{i u}^{u} \leq a_{i u}^{u} * \beta, \quad \forall u \in \mathscr{U}, i \in \mathscr{M} \cup \mathcal{S} .
$$

Equation (9) is a constraint of flow existence only on a valid access link, where $\beta$ is a big value, for example, $1 e+3$.

\section{Performance Evaluation}

We implemented our optimization model using optimization programming language (OPL) for IBM CPLEX Optimizer that searches feasible regions fast using branch-and-cut algorithm to obtain optimum value in our Mixed Integer Nonlinear Problem (MINLP). In addition, we developed a system level simulator (SLS) of a HetNet to get input data 


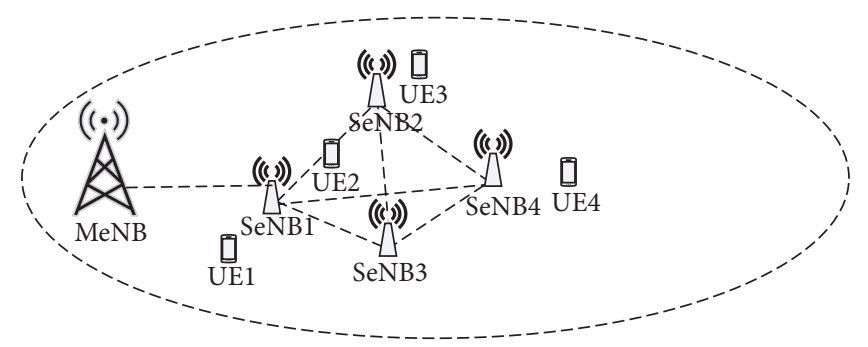

(a) Cluster at center

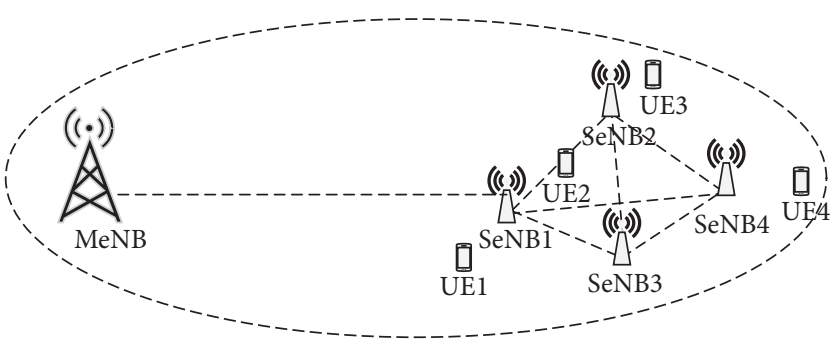

(b) Cluster at edge

FIGURE 6: mmWave-backhaul HetNet topology for preliminary experiments.

for the CPLEX such as access and backhaul link capacity, and interference vector. Simulation parameters used for the SLS are shown in Notations. We experiment with several HetNet topologies to investigate the optimal solution. Here we assume that backhaul links use the same mmWave band with the TDM and TDD methods.

4.1. Preliminary Experiment. First, we investigate UE throughput in a simple HetNet topology and small cell cluster at center and edge area as shown in Figure 6.

For the mmWave backhaul, total path loss is derived as the sum of the free space path loss $\left(\mathrm{PL}_{\text {free }}\right)$ and the signal attenuation from oxygen, vapour, and rain at distance $d$, as shown in the following equation:

$$
\mathrm{PL}_{(\mathrm{dB})}=\mathrm{PL}_{\mathrm{free}(\mathrm{dB})}+\mathrm{PL}_{d(\mathrm{~dB})} \text {. }
$$

The free space path loss is given by [23]

$$
\begin{aligned}
\mathrm{PL}_{\text {free }(\mathrm{dB})}= & 20 \log _{10}\left(f_{B H(\mathrm{MHz})}\right)+32.45 \\
& +20 \log _{10}\left(d_{(\mathrm{km})}\right) .
\end{aligned}
$$

Then, the attenuation at distance $d$ can be expressed as

$$
\mathrm{PL}_{d(\mathrm{~dB})}=d_{(\mathrm{km})}\left(L_{\mathrm{vap}}+L_{\mathrm{O}_{2}}+L_{R}\right)_{(\mathrm{dB} / \mathrm{km})},
$$

where $L_{\text {vap }}, L_{\mathrm{O}_{2}}$, and $L_{R}$ correspond to signal attenuation due to vapour water, oxygen [24], and rain [25], respectively. The rain attenuation $\lambda_{R}(\mathrm{~dB} / \mathrm{km})$ is obtained from the rain rate $R$ $(\mathrm{mm} / \mathrm{h})$ using the power-law relationship [25]:

$$
\lambda_{R}=k R^{\alpha} \text {. }
$$

4.1.1. Center Cluster. In Figure 6(a), a single small cell cluster is located at center of a macro cell, which includes 4 SeNBs and 4 UEs associated with one of the SeNBs for dual connectivity. In this topology, maximum link capacity from/to the MeNB or SeNB for UE is different according to the UE location. The UE1 has a downlink from only SeNB1 by $0.9 \mathrm{Mbps}$, UE2 has two $17.1 \mathrm{Mbps}$ links from SeNB1 and SeNB2, UE3 has downlinks from SeNB1 and SeNB2 with 0.9 and 39.6 Mbps, and UE4 has only SeNB4 with 0.9 Mbps. Maximum downlink rate from the MeNB is $39.6 \mathrm{Mbps}$, the same for all UEs in the center cluster. The backhaul links use $200 \mathrm{MHz}$ bandwidth at $60 \mathrm{GHz}$ and maximum
TABLE 1: UE throughput with RSRP/RSRQ based association.

\begin{tabular}{lccc}
\hline UE & Route & MeNB rate & SeNB rate \\
\hline UE1 & SeNB 1 & 15.05 & 0.9 \\
UE2 & SeNBs 1 and 2 & 9.5 & 6.48 \\
UE3 & SeNBs 1 and 2 & 0 & 20.59 \\
UE4 & SeNBs 1 and 4 & 15.05 & 0.9 \\
\hline
\end{tabular}

TABLE 2: UE throughput with traffic-aware SeNB addition.

\begin{tabular}{lccc}
\hline UE & Route & MeNB rate & SeNB rate \\
\hline UE1 & $*$ & 18.21 & 0 \\
UE2 & SeNB 1 & 4.75 & 13.5 \\
UE3 & SeNBs 1 and 2 & 0 & 39.6 \\
UE4 & SeNBs 1 and 4 & 16.63 & 0.9 \\
\hline
\end{tabular}

$*$ : No available multihop route.

256 QAM with 6.4 Bit-Per-Hertz (BPH) spectral efficiency. In this preliminary experiment, backhaul congestion will not happen.

Tables 1 and 2 show achievable flow rates on the MeNB and SeNB when using different SeNB addition approaches with fairness $\kappa=1 e-3$. Route column shows a route of a split bearer for dual connectivity. For example, the UE2 has dual flows from the MeNB directly and the SeNB2 via the SeNB1. MeNB and SeNB rate columns show achieved flow rates, Mbps, on each eNB. For total throughput, legacy approach based on RSRP/RSRQ fulfils $68.47 \mathrm{Mbps}$ for 4 UEs while total $93.59 \mathrm{Mbps}$ are achieved in the traffic-aware SeNB addition. In Figure 6(a), the UE2 can have SeNB1 or SeNB2 as a secondary eNB. In legacy approach, the MeNB configures the UE2 with the SeNB2 that has better RSRP/RSRQ than the SeNB1. Accordingly, the SeNB2 capacity is shared by UE2 and UE3 even though the UE3 has a higher MCS level than the UE2. On the other hand, the traffic-aware SeNB selection makes the UE2 associate with the SeNB1 instead of the SeNB2, which enables the UE3 to use fully the SeNB2.

4.1.2. Edge Cluster. In Figure 6(b), the small cell cluster is located at the edge of the macro cell. The UEs have different modulation orders from the macro cell according to their position compared to the center case; UE1 has maximum 39.6 Mbps, UE2 and UE3 have $34.3 \mathrm{Mbps}$, and UE4 has 


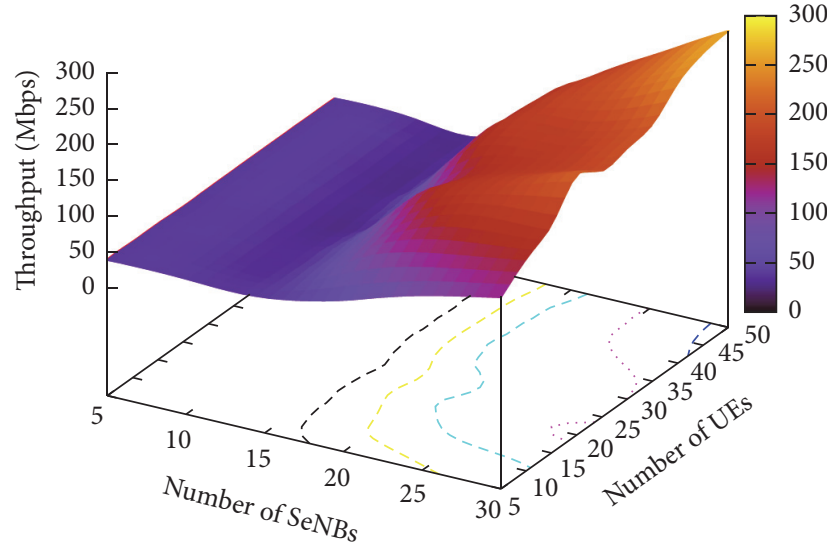

(a) Average throughput with fairness, $\kappa=1 e-6$

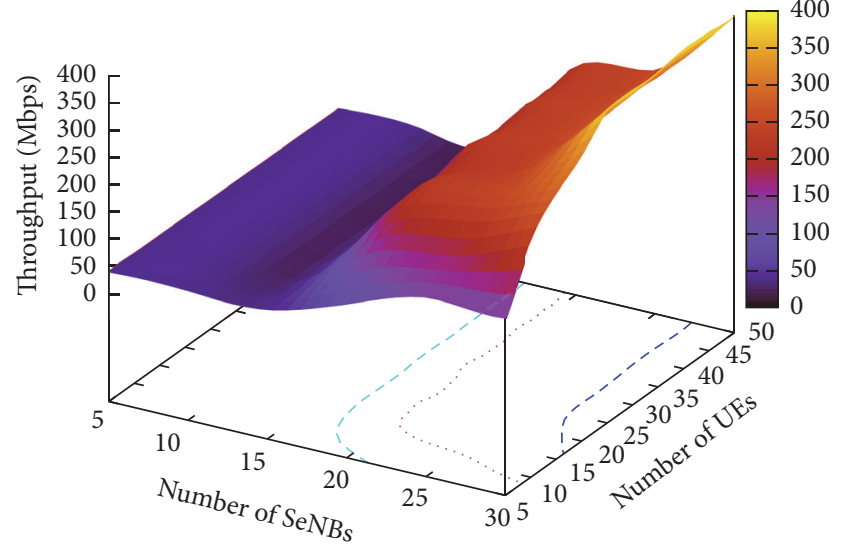

(b) Average throughput with fairness, $\kappa=1 e 6$

FIGURE 7: Aggregated throughput in mmWave-backhaul HetNet random topology.

TABLE 3: UE throughput fairness in the cell edge cluster.

\begin{tabular}{lcccc}
\hline \multirow{2}{*}{ UE } & \multicolumn{2}{c}{$\kappa=1 e 3$} & \multicolumn{2}{c}{$\kappa=1 e-3$} \\
& MeNB & SeNB & MeNB & SeNB \\
\hline UE1 & 39.6 & 0.9 & 12.67 & 0.9 \\
UE2 & 0 & 6.39 & 2.16 & 11.29 \\
UE3 & 0 & 39.6 & 0 & 13.46 \\
UE4 & 0 & 0.9 & 12.61 & 0.9 \\
\hline
\end{tabular}

12.6 Mbps from the macro cell without overhead. Access link capacity to SeNBs in the edge cluster is the same as the center cluster.

With the same $\kappa=1 e-3$, the total throughput decreases almost $40 \mathrm{Mbps}$ compared to the center cluster case as can be seen in Table 3. Total throughput of UEs is 87.39 and $53.99 \mathrm{Mbps}$ for $\kappa=1 e 3$ and $1 e-3$, respectively. The throughput difference between the fairness, $\kappa=1 e-3$, and $1 e+3$ values is about $40 \%$. The fairness can affect the aggregated UE throughput significantly; the throughput can be varying more than $40 \%$ with less than $1 e-3$ or more than $1 e+3 \kappa$ values.

Jain's fairness index $(J)$ of UE throughput shows 0.58 and 0.99 with those two $\kappa$ values $(J=[0,1]$ and fair as $J$ is close to 1$)$. Difference of the two Jain's indices is about 0.4 :

$$
J\left(x_{1}, x_{2}, \ldots, x_{n}\right)=\frac{\left(\sum_{i=1}^{n} x_{i}\right)^{2}}{n \cdot \sum_{i=1}^{n} x_{i}^{2}}
$$

4.1.3. Random Cluster. Next, we investigate average aggregated UE throughput using random topologies with varying number of UEs and SeNBs. Those UEs and SeNBs drop within a single sector of a macro cell. Figure 7 shows the aggregated UE throughput with maximum 30 SeNBs and 50 UEs. The throughput is investigated with different values of $\kappa=1 e 6$ and $1 e-6$ for different fairness levels, as shown in Figures 7(a) and 7(b).
With low number of SeNBs, consistent throughput is observed in both figures since the UEs have limited opportunity to access SeNBs. However, throughput increases fast as the number of SeNBs and UEs increases, especially for the $\kappa=1 e 6$ case. Maximum throughput of the $\kappa=1 e 6$ is higher than the $\kappa=1 e-6$ by more than $100 \mathrm{Mbps}$ since proportional fairness with the higher $\kappa$ value can achieve more throughput. The Jain index is 0.83 and 0.42 with $\kappa=1 e-6$ and $1 e 6$, respectively. With the previous values of $\kappa=1 e-3$ and $1 e+3$, difference of Jain's indices was less than 0.4 as the Jain index of the $\kappa=1 e-3$ is less than 0.83 .

4.2. SeNB Selection Algorithm. SeNB selection for dual connectivity and flow maximization is MINLP which is NPhard mostly. Thus, IBM CPLEX solvers spend much time to find optimal solution as the number of SeNBs and UEs increases. For example, the optimization problem with 100 SeNBs and 100 UEs took 98 seconds for a particular topology; calculation complexity depends on the topology and actual run time is more than the calculation time. To reduce the calculation time, we propose a simple heuristic algorithm for the SeNB selection and multihop routing in dual connectivity procedure which can perform close to optimal results of the IBM CPLEX in terms of UE throughput and fairness.

To develop an algorithm maximizing UE throughput, first we decompose the problem into two scheduling problems in MeNB and SeNB as in (15) since PRB allocation in the MeNB and SeNB can be conducted independently which is a common assumption in dual connectivity due to backhaul latency [26, 27]. Thus, our algorithm focuses on maximizing aggregated UE throughput in SeNBs (see (16)) with SeNB and multihop backhaul selection, which are eventually determined by available resources in access and backhaul networks:

$$
\begin{aligned}
& \max U\left(F^{u}\right)=\max U\left(F_{i}^{u}+F_{j}^{u}\right), \\
& \quad i \in \mathscr{M}, j \in \mathcal{S}, \\
& \arg \max _{i} U\left(F_{i}^{u}\right)=\gamma+\kappa \sum_{i} \sum_{u} F_{i}^{u}, \quad i \in \mathcal{S} .
\end{aligned}
$$




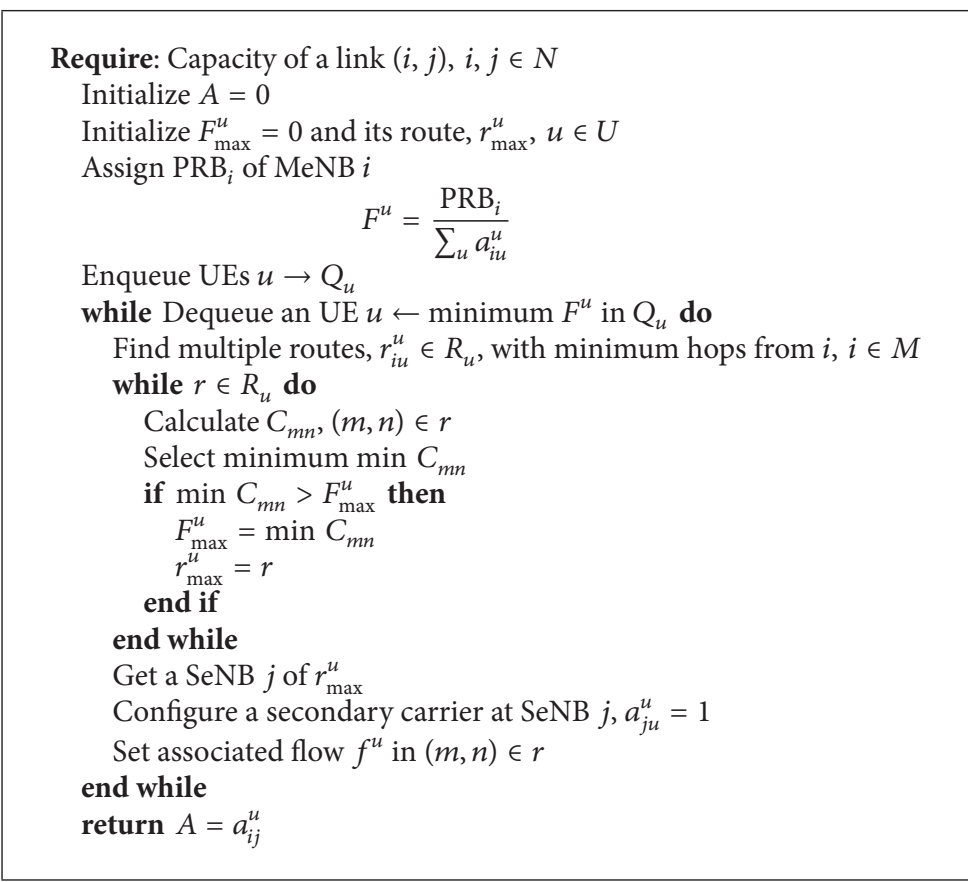

Algorithm 1: SeNB and multihop backhaul selection for dual connectivity.

Algorithm 1 describes details of the SeNB and backhaul selection algorithm for dual connectivity. Given the link capacity information, the algorithm decides the vector $a_{i j}^{u}$ of SeNB association for each UE. First, MeNB PRB allocation is preprocessed before entering SeNB selection because UEs have a connection to the MeNB in advance before adding an SeNB for a secondary connection. Once the PRBs are assigned for UEs, the algorithm enqueues UEs in $Q_{u}$ with ascending order of UE utility. Then, the algorithm seeks multiple routes from an MeNB to UE with minimum hops instead of considering all possible routes using a shortest path algorithm. For each route, the algorithm calculates achievable flow rate by finding a minimum capacity link, that is, a bottleneck link. The link capacity, $C_{m n}$, is calculated by dividing link capacity by a number of associated UE flows. For example, a $(m, n)$ link with $100 \mathrm{Mbps}$ can provide $10 \mathrm{Mbps}$ for new UE if 9 UE flows are assigned. Thereafter, the algorithm selects a maximum capacity route and an SeNB that is located on the route. Finally, the algorithm returns the association vector, $a_{i j}^{u}$, for all UEs. The algorithm considers only the routes via SeNBs that have valid access links to UEs within coverage. Complexity of this algorithm can be $O\left(U|S|^{2}+U L\right)$ where Dijkstra's algorithm, $O\left(|S|^{2}\right)$, is executed for multihop routing of each UE.

For evaluation, the proposed algorithm is applied to the center cluster topology shown in Figure 6(a). Table 4 shows simulation results of achieved throughput per UE. Since all UEs have similar MCS levels in the MeNB, each UE is evenly assigned by about $9.5 \mathrm{Mbps}$ from the MeNB. But the UEs have different data rate in the SeNBs according to traffic load and MCS levels. Our algorithm achieves total 68.1 Mbps which is less than the optimal solution in Table 2 but comparable
TABLE 4: Throughput with the proposed algorihtm in center cluster.

\begin{tabular}{lccc}
\hline UE & Route & MeNB rate & SeNB rate \\
\hline UE1 & SeNB 1 & 9.5 & 0.9 \\
UE2 & SeNBs 1 and 2 & 9.5 & 8.5 \\
UE3 & SeNBs 1 and 2 & 9.5 & 19.8 \\
UE4 & SeNBs 1 and 4 & 9.5 & 0.9 \\
\hline
\end{tabular}

TABLE 5: Throughput with the proposed algorihtm in edge cluster.

\begin{tabular}{lccc}
\hline UE & Route & MeNB rate & SeNB rate \\
\hline UE1 & SeNB 1 & 9.6 & 0.9 \\
UE2 & SeNBs 1 and 2 & 8.5 & 8.5 \\
UE3 & SeNBs 1 and 2 & 8.5 & 19.8 \\
UE4 & SeNBs 1 and 4 & 3.1 & 0.9 \\
\hline
\end{tabular}

to the legacy approach. The fairness is similarly supported; Jain's fairness index is 0.83 in our algorithm while the CPLEX solution was 0.86 .

Table 5 shows UE throughput in the edge cluster of Figure 6(b) when using the proposed algorithm. Total throughput is $59.7 \mathrm{Mbps}$ which is higher than the throughput with $\kappa=1 e-3,53.6 \mathrm{Mbps}$, but less than $\kappa=1 e+3$, 87.4 Mbps. In terms of fairness, Jain's fairness index of the proposed scheme is 0.73 which is also less than the $\kappa=1 e-3$ case but more than $\kappa=1 e+3$.

4.3. Comparison Study in Random Topology. In this section, we compare aggregated UE throughput of optimal and proposed solutions with varying number of SeNBs and UEs which drop uniformly within a single macro cell sector. With 


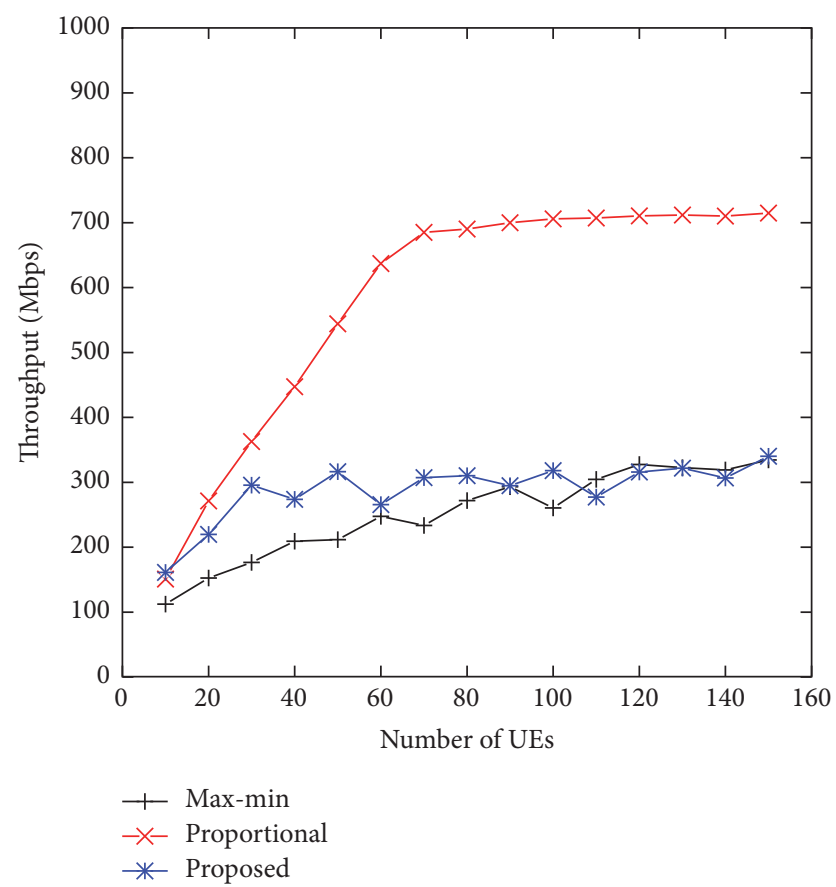

Figure 8: Aggregated throughput comparison in 30 SeNBs (access link: $10 \mathrm{MHz}$ and Backhaul link: $200 \mathrm{MHz}$ ).

50 random topologies, we derived average values from IBM CPLEX outcomes with $\kappa=1 e 9$ and $1 e-9$ for proportional and max-min fairness (we abuse the terms of max-min and proportional fairness for comparison (but not for fairness), which indicates lower and upper bounds, resp., set by maxweight and max-min scheduling). Jain's fairness indices of the proportional and max-min fairness are approximately 0.2 and 0.6 , respectively. Also, we obtain average throughput of our algorithm with the same number of random topologies to compare with throughput bounds given by the CPLEX solver.

First, with given bandwidth for access and backhaul links, 10 and $200 \mathrm{MHz}$ of 30 SeNBs, average aggregated throughput of varying number of UEs is shown in Figure 8. Throughput increases by the number of UEs in all cases. The throughput is saturated in particular number of UEs which is different to each solution; proportional scheme reaches maximum throughput around 70 UEs, max-min scheme increases slightly up to $160 \mathrm{UEs}$, and our algorithm is saturated near 60 UEs. Proportional case becomes saturated earlier than the max-min case since it utilizes link capacity aggressively without fairness consideration among UEs. Our algorithm also uses link capacity fully but evenly share with other UEs, which limits the throughput near the max-min throughput. Jain's fairness indices of the optimal solutions are 0.62 and 0.25 . UEs can have dual connectivity or not according to the random topology, which degrades the fairness index compared to the preliminary results.

The proposed algorithm shows higher throughput than the max-min approach with small number of UEs, but its throughput becomes similar with the max-min approach as the number of UEs increases. The proposed algorithm achieves more throughput by assigning all SeNB PRBs to

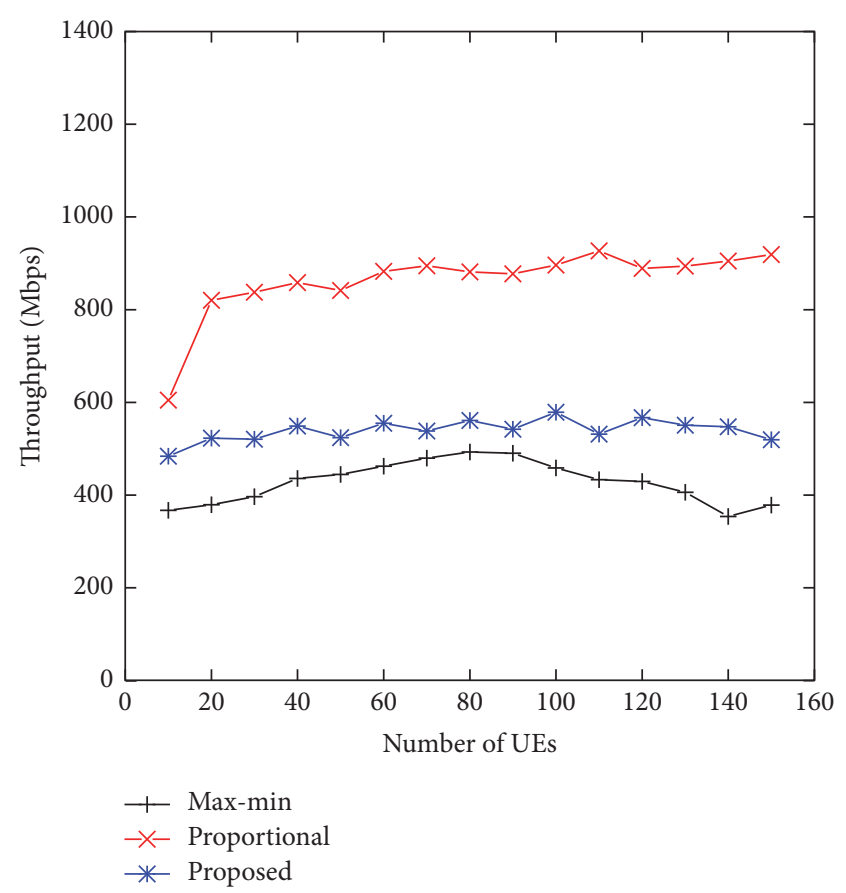

FIGURE 9: Aggregated throughput comparison with more UE traffic $(20 \mathrm{MHz}$ and $2 \times 2 \mathrm{MIMO})$.

even small number of UEs while the max-min approach underutilizes the access links with the small number of UEs due to fairness. Accordingly, the proposed algorithm can achieve similar throughput with the max-min approach in congested situation and better throughput in uncongested situation with lower fairness.

In previous experiment, maximum capacity of an access link $(10 \mathrm{MHz})$ is relatively smaller than the backhaul link $(200 \mathrm{MHz})$. Thus, more UE traffic load is generated by increased access bandwidth, $20 \mathrm{MHz}$, and $2 \times 2 \mathrm{MIMO}$ antenna in the 30 SeNBs. Figure 9 shows that the aggregated UE throughput increases only about $100 \mathrm{Mbps}$ even though the access network capacity increases almost 4 times the previous configuration. Thus, we can conjecture that the UE traffic is congested in the backhaul links with throughput values in Figure 9. All approaches are saturated earlier even with small number of UEs due to increased access data rate. It is notable that the throughput decreases slightly in high number of UEs due to overhead. The proposed scheme also performs similarly with the max-min approach like in previous experiment.

There might be two reasons for the backhaul congestion; one is limited number of backhauls to an MeNB and the other is backhaul bandwidth. A backhaul link capacity with even $200 \mathrm{MHz}$ bandwidth can be limited by adjacent links for TDM operation. In Figure 10, 50 SeNBs are deployed to afford the increased mobile traffic. The proportional case gains about $50 \%$ of previous throughput, more or less $400 \mathrm{Mbps}$, and others achieve $25 \%$ gain, about $100 \mathrm{Mbps}$ more than before as the newly added 20 SeNBs contribute to increasing the number of backhauls to the MeNB. 


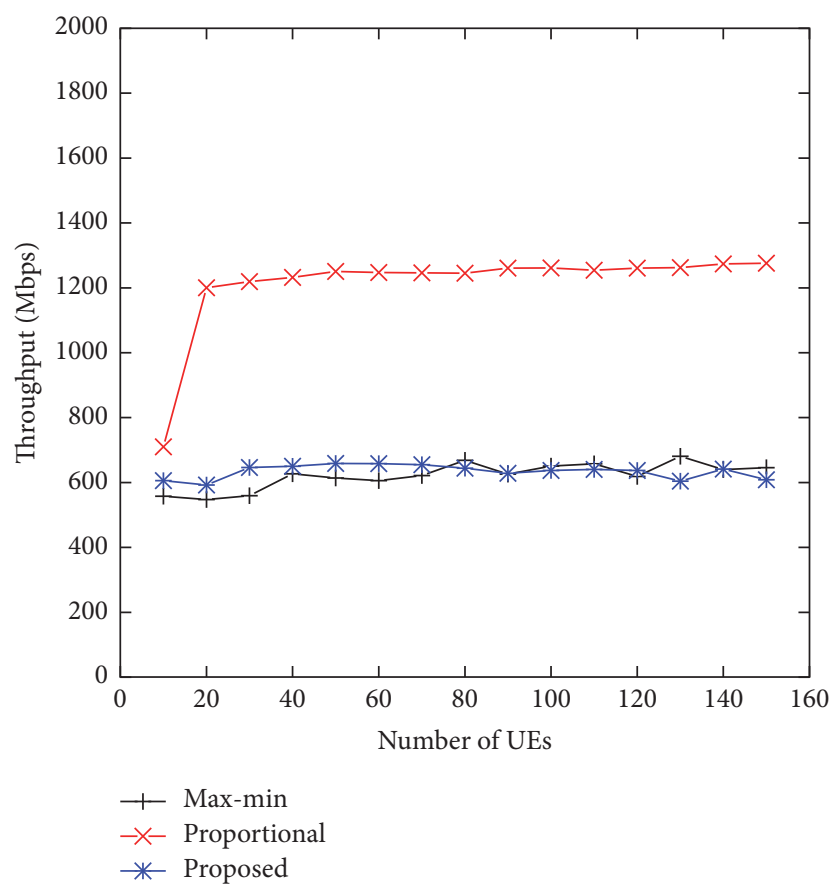

FIgURE 10: Aggregated throughput with more UE traffic $(20 \mathrm{MHz}$ and $2 \times 2$ MIMO) in 50 SeNBs.

Next, we increase backhaul bandwidth itself up to $400 \mathrm{MHz}$ with $30 \mathrm{SeNBs}$ to see total throughput change. Figure 11 depicts that the proportional scheme shows enhanced throughput about $1500 \mathrm{Mbps}$, almost $100 \%$ gain. In case of the max-min, however, throughput before 100 UEs is comparable to the results with $200 \mathrm{MHz}$ backhaul. After 100 UEs, throughput with more backhaul capacity is notably higher than the previous one. Proposed scheme also gains more throughput about $200 \mathrm{Mbps}$ with the increased backhaul bandwidth. From above observation, we can use more bandwidth, such as $1 \mathrm{GHz}$ and $2 \mathrm{GHz}$, dynamically to cope with more mobile traffic with massive antenna, for example, $4 \times 4$ MIMO recently commercialized for smart phones and Carrier Aggregation in access networks.

In conclusion, our algorithm performs almost half of peak throughput of optimal solution for the proportional fairness while it was comparable with the max-min fairness according to several simulations.

\section{Related Works}

5.1. Small Cell Deployment. Ultradense Network (UDN) with small cells is one of the key technologies for 5G networks as mentioned in METIS project [1] and heterogeneous small cell networks have been popularly researched as in many research publications and EU/US projects [3]. In the HetNets with dense small cell deployment, interference management mechanisms are intensively explored in the colayer (i.e., cotier) and cross-layer (i.e., cross-tier) channel deployment for macro and small cells in the HetNet [28-34].

In standard, 3GPP Rel-11 started studying about HetNet mobility in the colayer and cross-layer channel deployment

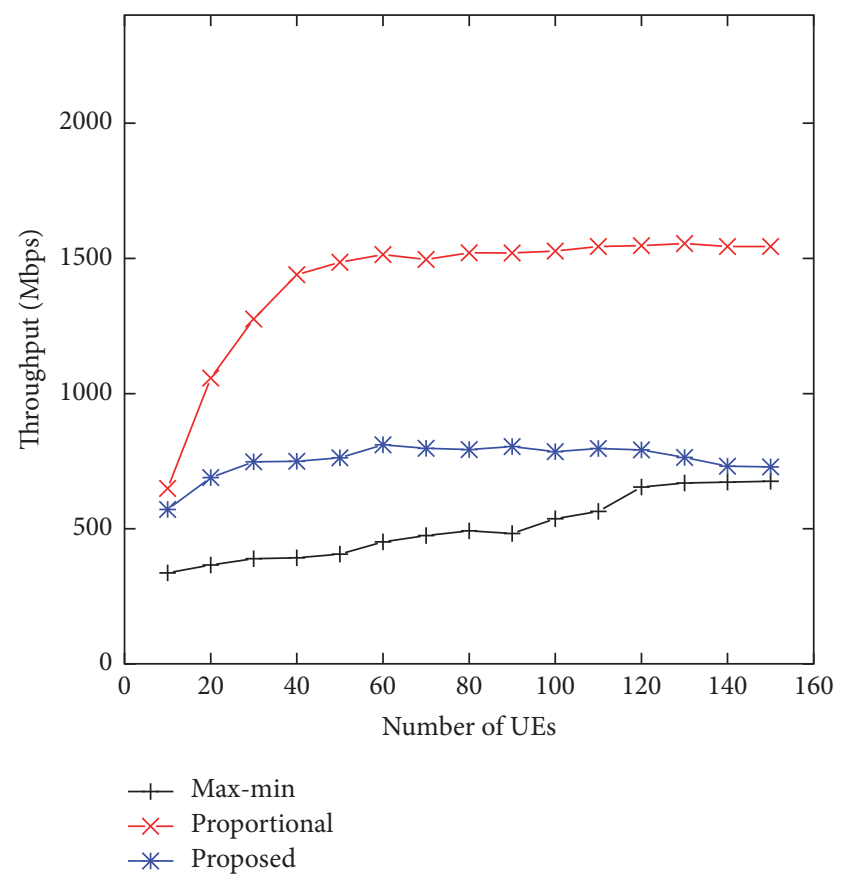

FIgURE 11: Aggregated throughput comparison in $30 \mathrm{SeNBs}$ (access link: $20 \mathrm{MHz}$ and Backhaul link: $400 \mathrm{MHz}$ ).

[15]. In 3GPP Rel-12, study about small cell enhancement (SCE) introduced features of mobility enhancement, signalling overhead reduction, RRC diversity, and dual connectivity [26]. The dual connectivity can achieve higher data rate than a single connection and separate a control and data path. Also, the dual connectivity provides robust mobility among small cells with an MeNB as an anchor point, which prevents frequent handover procedures [35]. In [36], traffic steering and TDD configuration algorithms have been proposed for dual connectivity in dynamic TDD-based HetNets.

5.2. mmWave Communication. There has been much effort to use the mmWave band from $30 \mathrm{GHz}$ to $300 \mathrm{GHz}$ due to scarcity of available bands in microwave spectrum. $60 \mathrm{GHz}$ band and E-bands $(7176 \mathrm{GHz}$ and $8186 \mathrm{GHz})$ have a huge amount of available bandwidth, 5-7 GHz over the world.

WPAN or WLAN standards such as IEEE 802.15.3 Task Group 3c (TG3c) [4] and IEEE 802.11ad [5] are already published, which specifies physical MAC layer protocols for mmWave communications. Those standards typically adopt hybrid Time Division Multiple Access (TDMA) scheme for medium access control (MAC) $[4,5,37,38]$. For instance, in the IEEE 802.11ad, a hybrid multiple access that consists of Carrier Sensing Multiple Access/Collision Avoidance (CSMA/CA) and TDMA is mixed flexibly for spectral efficiency and quality of service (QoS). Also, IEEE 802.15.3c defines Contention Access Period (CAP) and the Channel Time Allocation Period (CTAP). During the CAP, devices have contention to send transmission requests to the a piconet controller (PNC) by the CSMA/CA. Then data transmissions among devices are scheduled during the CTAP using TDMA. 
There are many literatures to investigate MAC protocols for mmWave communication. Based on IEEE $802.11 \mathrm{ad}$, Chen et al. [39] proposed a spatial reuse strategy for concurrent transmissions with directional antennas. Son et al. [40] proposed a frame-based directive MAC (FDMAC) protocol which is a centralized scheduling algorithm for the PNC based on Greedy Coloring providing multiple concurrent transmissions.

Singh et al. [41] proposed a memory-guided directional MAC (MDMAC), as a fully distributed MAC protocol, which achieves approximate TDM scheduling for wireless meshes using memory about transmission success and fail. Another distributed algorithm, directional-to-directional MAC (DtDMAC) [42], uses an exponential backoff procedure for asynchronous operation. In both cases, a deafness effect is alleviated by a Markov state transition diagram.

Recently, this mmWave attracts attention in areas of wireless cellular networks or mesh networks as 5G communication. Rappaport et al. [7] conducted measurement campaign in New York City on the $28 \mathrm{GHz}$. There have been also channel measurements for mmWave cellular in the $28 \mathrm{GHz}$, $38 \mathrm{GHz}$, and $73 \mathrm{GHz}$ bands [8, 9]. Reference [43] shows architecture and performance of mmWave-based dense small cell networks as $5 \mathrm{G}$ networks. References $[17,19]$ introduce a heterogeneous small cell network using mmWave communication for access and backhaul networks where multihop routing from UEs to MeNBs is supported in the backhaul meshes. For radio resource allocation for the access and backhaul links, [19] proposes a frame-based TDD and TDM scheme, which increases spatial reuse of mmWave bands based on small cell clusters. Also, Taori and Sridharan [20] proposed a TDM-based scheduling for in-band access and backhaul links to support point-to-multipoint and non-LoS communication. Reference [44] compares static or dynamic algorithms for multihop routing in backhaul networks using a node and path centric model. Reference [45] proposes beam alignment and subspace sampling to overcome impairment at outdoor access and backhaul links. Reference [46] introduced energy- and spectral efficiency-aware cell association mechanism in $60 \mathrm{GHz}$ wireless backhaul links, which solves a multiobjective problem to maximize throughput while minimizing energy consumption.

\section{Conclusion}

In this paper, we establish an optimization model for dual connectivity in small cell HetNets with mmWave backhauls. We also propose a simple heuristic algorithm for the dual connectivity to replace commercial optimizer. Using IBM CPLEX, we explore upper and lower bounds of aggregated UE throughput using different fairness weights in random HetNet topologies. Based on that, we compare performance of our algorithm. According to simulation results, dense SeNBs and large mmWave bandwidth improve network throughput by increasing access and backhaul link capacity. In the varying number of SeNBs and backhaul bandwidth, our algorithm achieves comparable throughput with the lower bound of the optimizer's solution. In future work, we study about SeNB on and off and energy efficient routing for power saving in the HetNet with mmWave backhauls.

\section{Notations}

Parameters and Variables in the Model

$N_{\text {PRB }}$ : Maximum number of PRBs of an eNB

$C_{i j}, C_{l}$ : Maximum capacity of a link $(i, j)$ or $l$

$f_{i j}^{u}: \quad$ Flow of UE $u$ on link $(i, j)$

$F^{u}$ : $\quad$ Flow of UE $u$

$\gamma: \quad$ Minimum required UE throughput

$\mathscr{I}: \quad$ Set of interference links

$\mathscr{L}_{\mathscr{A}}:$ Set of access links

$\mathscr{L}_{\mathscr{B}}$ : Set of backhaul links

$\mathscr{M}: \quad$ Set of macro eNBs (MeNBs)

$\mathcal{S}: \quad$ Set of small eNBs (SeNBs)

$\mathcal{N}: \quad$ Set of eNBs

$\mathrm{PRB}_{l}$ : Number of PRBs at link $l$

$\mathcal{U}: \quad$ Set of UEs

$a_{i j}^{u}$ : Indicator if UE $u$ uses access link $(i, j)$.

\section{Simulation Parameters}

$\begin{array}{ll}\text { MeNB radius: } & 500 \mathrm{~m} \\ \text { Min ISD of SeNB: } & 20 \mathrm{~m} \\ \text { Min ISD between MeNB and SeNB: } 105 \mathrm{~m} \\ \text { Min distance between eNB and UE: } & \text { MeNB } 35 \mathrm{~m} \text { and SeNB } \\ & 5 \mathrm{~m} \\ \text { Bandwidth: } & 10 \mathrm{MHz} \\ \text { Carrier frequency: } & \text { MeNB } 2 \mathrm{GHz} \text { and } \\ & \text { SeNB } 2.6 \mathrm{GHz} \\ \text { eNB Tx power: } & \text { MeNB } 46 \mathrm{dBm} \text { and } \\ & \text { SeNB } 30 \mathrm{dBm} \\ \text { UE Tx power: } & 23 \mathrm{dBm} \\ \text { Antenna gain: } & \text { MeNB } 15 \mathrm{dBi} \text { and } \\ & \text { SeNB } 5 \mathrm{dBi} \\ \text { Noise figure: } & 7 \mathrm{~dB} \\ \text { Shadowing standard deviation: } & \text { MeNB } 8 \mathrm{~dB} \text { and SeNB } \\ & 10 \mathrm{~dB} \\ \text { Multipath delay profile: } & \text { Typical Urban } \\ \text { UE speed: } & \text { Static } \\ \text { Path loss: } & \text { MeNB } 128.1+ \\ & 37.6 \log 10(r) \text { and } \\ & \text { SeNB } 140.7+ \\ & 36.7 \log 10(r) .\end{array}$

\section{Competing Interests}

The author declares that there is no conflict of interests regarding the publication of this paper.

\section{Acknowledgments}

This research was supported by the Gachon University Research Fund GCU-2015-0044 of 2015. 


\section{References}

[1] A. Osseiran, F. Boccardi, V. Braun et al., "Scenarios for 5G mobile and wireless communications: the vision of the METIS project," IEEE Communications Magazine, vol. 52, no. 5, pp. 2635, 2014.

[2] D. Soldani and A. Manzalini, "Horizon 2020 and beyond: on the $5 \mathrm{G}$ operating system for a true digital society," IEEE Vehicular Technology Magazine, vol. 10, no. 1, pp. 32-42, 2015.

[3] S. Yunas, M. Valkama, and J. Niemelý, "Spectral and energy efficiency of ultra-dense networks under different deployment strategies," IEEE Communications Magazine, vol. 53, no. 1, pp. 90-100, 2015.

[4] 802.15.3c-2009-IEEE Standard for Information TechnologyLocal and Metropolitan Area Networks-Specific Requirements-Part 15.3: Amendment 2: Millimeter-Wave-Based Alternative Physical Layer Extension.

[5] "Part11: Wireless LAN Medium Acess Control (MAC) and Physical Layer (PHY) Specifications C Amendment 5: Enhancements for Very High Throughput in the $60 \mathrm{GHz}$ Band. IEEE P802.11ad/D1.0," 2010.

[6] Wireless Gigabit Alliance (WiGig), 2016, http://www.wi-fi.org/ discover-wi-fi/wi-fi-certified-wigig.

[7] T. S. Rappaport, S. Sun, R. Mayzus et al., "Millimeter wave mobile communications for 5G cellular: it will work!," IEEE Access, vol. 1, pp. 335-349, 2013.

[8] S. Sun, G. R. Maccartney, M. K. Samimi, S. Nie, and T. S. Rappaport, "Millimeter wave multi-beam antenna combining for 5G cellular link improvement in New York City," in Proceedings of the 1st IEEE International Conference on Communications (ICC '14), pp. 5468-5473, IEEE, Sydney, Australia, June 2014.

[9] G. R. Maccartney Jr. and T. S. Rappaport, "73 GHz millimeter wave propagation measurements for outdoor urban mobile and backhaul communications in New York City," in Proceedings of the 1st IEEE International Conference on Communications (ICC '14), pp. 4862-4867, Sydney, Australia, June 2014.

[10] A. Ghosh, R. Ratasuk, B. Mondal, N. Mangalvedhe, and T. Thomas, "LTE-advanced: next-generation wireless broadband technology," IEEE Wireless Communications, vol. 17, no. 3, pp. $10-22,2010$.

[11] 3GPP, "Small cell enhancements for E-UTRA and E-UTRANphysical layer aspects," Tech. Rep. TR 36.872, 3GPP, 2013.

[12] Z. Shen, A. Papasakellariou, J. Montojo, D. Gerstenberger, and F. Xu, "Overview of 3GPP LTE-advanced carrier aggregation for $4 \mathrm{G}$ wireless communications," IEEE Communications Magazine, vol. 50, no. 2, pp. 122-130, 2012.

[13] 3GPP, "TR 36.932 scenarios and requirements for small cell enhancements for e-utra and e-utran," Tech. Rep., 2012.

[14] 3GPP, "TR 36.842 study on small cell enhancements for e-utra and eutran; Higher layer aspects," Tech. Rep., 3GPP, 2013.

[15] 3GPP, "TR 36.839 mobility enhancements in heterogeneous networks," Tech. Rep., 3GPP, 2012.

[16] J. F. Monserrat, H. Droste, Ó. Bulakci et al., "Rethinking the mobile and wireless network architecture: the METIS research into 5G," in Proceedings of the European Conference on Networks and Communications (EuCNC '14), pp. 1-5, Bologna, Italy, June 2014.

[17] C. Dehos, J. L. Gonzýlez, A. De Domenico, D. Ktýnas, and L. Dussopt, "Millimeter-wave access and backhauling: the solution to the exponential data traffic increase in $5 \mathrm{G}$ mobile communications systems?" IEEE Communications Magazine, vol. 52, no. 9, pp. 88-95, 2014.
[18] ECMC TC48, "High rate $60 \mathrm{GHz}$ PHY, MAC and HDMI PAL," ECMA Standard 387, 2008.

[19] P. Wang, Y. Li, L. Song, and B. Vucetic, "Multi-gigabit millimeter wave wireless communications for 5G: from fixed access to cellular networks," IEEE Communications Magazine, vol. 53, no. 1, pp. 168-178, 2015.

[20] R. Taori and A. Sridharan, "Point-to-multipoint in-band mmwave backhaul for $5 \mathrm{G}$ networks," IEEE Communications Magazine, vol. 53, no. 1, pp. 195-201, 2015.

[21] Qualcomm, "Rpa160054 considerations on 5g relay requirements," Tech. Rep., 2016.

[22] Samsung, "Wireless relay at mmwave frequency for $5 \mathrm{~g}$ new radio interface," Tech. Rep. R1-162187, 2016.

[23] J. D. Parsons and P. J. D. Parsons, The Mobile Radio Propagation Channel, 1992.

[24] ITU, "Recommendation ITU-R p.676-5. attenuation by atmospheric gases," Tech. Rep., International Telecommunication Union (ITU), Geneva, Switzerland, 2013.

[25] ITU, "Recommendation ITU-R p.838-3. Specific attenuation model for rain for use in prediction methods," Tech. Rep., ITU, 2013.

[26] D. Astely, E. Dahlman, G. Fodor, S. Parkvall, and J. Sachs, "LTE release 12 and beyond [Accepted From Open Call]," IEEE Communications Magazine, vol. 51, no. 7, pp. 154-160, 2013.

[27] 3GPP, “TS 36.300 evolved universal terrestrial radio access (EUTRA) and evolved universal terrestrial radio access network (E-UTRAN); overall description; stage 2," Tech. Rep., 3GPP, 2016.

[28] H. Claussen, "Performance of macro- and co-channel femtocells in a hierarchical cell structure," in Proceedings of the 18th Annual IEEE International Symposium on Personal, Indoor and Mobile Radio Communications (PIMRC '07), pp. 1-5, Athens, Greece, September 2007.

[29] H. Claussen, "Co-channel operation of macro- and femtocells in a hierarchical cell structure," International Journal of Wireless Information Networks, vol. 15, no. 3, pp. 137-147, 2008.

[30] V. Chandrasekhar, J. G. Andrews, and A. Gatherer, "Femtocell networks: a survey," IEEE Communications Magazine, vol. 46, no. 9, pp. 59-67, 2008.

[31] Y. Peng and F. Qin, "Exploring Het-Net in LTE-advanced system: interference mitigation and performance improvement in Macro-Pico scenario," in Proceedings of the IEEE International Conference on Communications Workshops (ICC '11), pp. 1-5, IEEE, Kyoto, Japan, June 2011.

[32] C. Na, X. Hou, and H. Jiang, "Interference alignment based dynamic TDD for small cells," in Proceedings of the IEEE Globecom Workshops, pp. 700-705, December 2014.

[33] N. Saquib, E. Hossain, L. B. Le, and D. I. Kim, "Interference management in OFDMA femtocell networks: issues and approaches," IEEE Wireless Communications, vol. 19, no. 3, pp. 86-95, 2012.

[34] G. Vivier, M. Kamoun, Z. Becvar, E. De Marinis, Y. Lostanlen, and A. Widiawan, "Femtocells for next-G wireless systems: the FREEDOM approach," in Proceedings of the Future Network and Mobile Summit, pp. 1-9, June 2010.

[35] K. I. Pedersen, P.-H. Michaelsen, C. Rosa, and S. Barbera, "Mobility enhancements for LTE-advanced multilayer networks with inter-site carrier aggregation," IEEE Communications Magazine, vol. 51, no. 5, pp. 64-71, 2013.

[36] W. Kim, "Adaptive resource scheduling for dual connectivity in heterogeneous IoT cellular networks," International Journal of 
Distributed Sensor Networks, vol. 2016, Article ID 6036952, 13 pages, 2016.

[37] X. An and R. Hekmat, "Directional MAC protocol for millimeter wave based wireless personal area networks," in Proceedings of the IEEE 67th Vehicular Technology Conference-Spring (VTC '08), pp. 1636-1640, May 2008.

[38] C.-W. Pyo, F. Kojima, J. Wang, H. Harada, and S. Kato, "Mac enhancement for high speed communications in the 802.15.3c mmwave wpan," Wireless Personal Communications, vol. 51, no. 4, pp. 825-841, 2009.

[39] Q. Chen, X. Peng, J. Yang, and F. Chin, "Spatial reuse strategy in mmWave WPANs with directional antennas," in Proceedings of the IEEE Global Communications Conference (GLOBECOM '12), pp. 5392-5397, Anaheim, Calif, USA, December 2012.

[40] I. K. Son, S. Mao, M. X. Gong, and Y. Li, "On frame-based scheduling for directional mmWave WPANs," in Proceedings of the IEEE Conference on Computer Communications (INFOCOM '12), pp. 2149-2157, IEEE, Orlando, Fla, USA, March 2012.

[41] S. Singh, R. Mudumbai, and U. Madhow, "Distributed coordination with deaf neighbors: efficient medium access for $60 \mathrm{GHz}$ mesh networks," in Proceedings of the IEEE Conference on Computer Communications (INFOCOM '10), pp. 1-9, March 2010 .

[42] E. Shihab, L. Cai, and J. Pan, "A distributed asynchronous directional-to-directional MAC protocol for wireless ad hoc networks," IEEE Transactions on Vehicular Technology, vol. 58, no. 9, pp. 5124-5134, 2009.

[43] A. Ghosh, T. A. Thomas, M. C. Cudak et al., "Millimeterwave enhanced local area systems: a high-data-rate approach for future wireless networks," IEEE Journal on Selected Areas in Communications, vol. 32, no. 6, pp. 1152-1163, 2014.

[44] M. Shariat, M. Dianati, K. Seppänen, T. Suihko, J. Putkonen, and V. Frascolla, "Enabling wireless backhauling for next generation mmWave networks," in Proceedings of the European Conference on Networks and Communications (EuCNC '15), pp. 164-168, Paris, France, July 2015.

[45] S. Hur, T. Kim, D. J. Love, J. V. Krogmeier, T. A. Thomas, and A. Ghosh, "Millimeter wave beamforming for wireless backhaul and access in small cell networks," IEEE Transactions on Communications, vol. 61, no. 10, pp. 4391-4403, 2013.

[46] A. Mesodiakaki, F. Adelantado, L. Alonso, and C. Verikoukis, "Energy-efficient user association in cognitive heterogeneous networks," IEEE Communications Magazine, vol. 52, no. 7, pp. 22-29, 2014. 

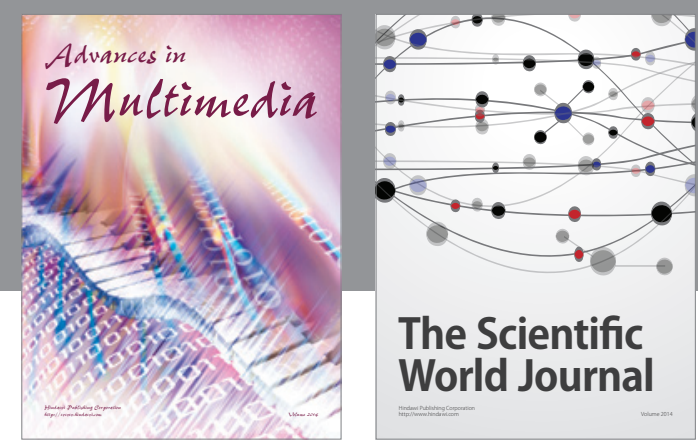

The Scientific World Journal
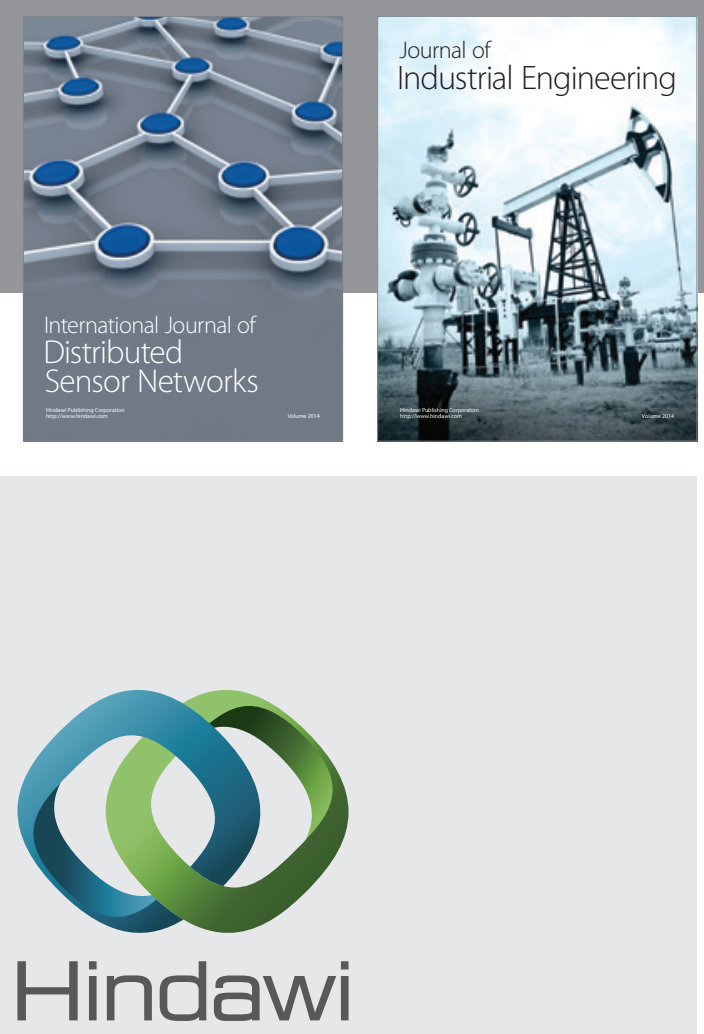

Submit your manuscripts at

http://www.hindawi.com

\section{Computer Networks} and Communications
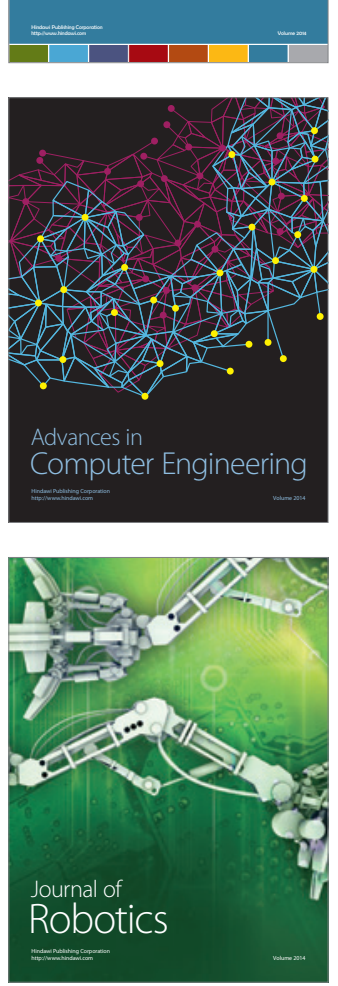
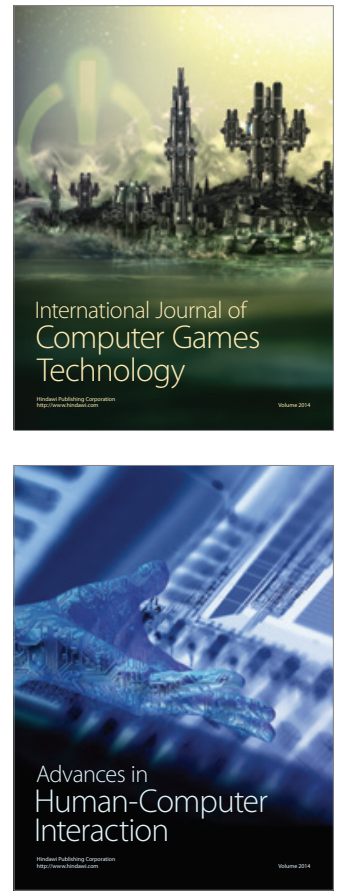
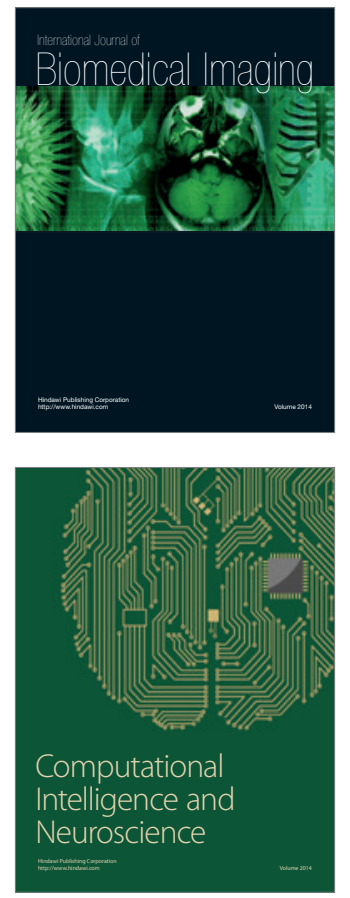
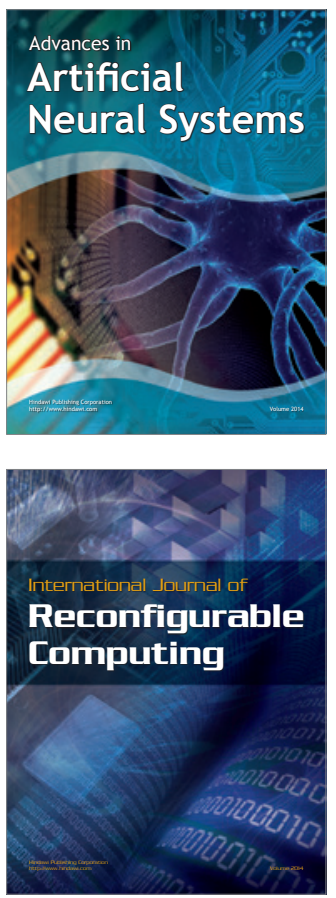
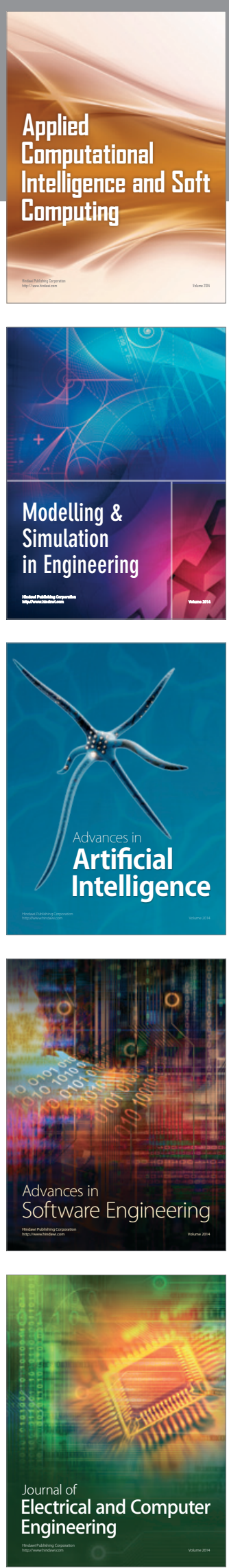